\title{
The solutions used for long wave thermographic cameras designed for observing elements in SMD housings
}

\author{
Krzysztof Dziarski ${ }^{1, *}$, Joanna Parzych $^{1}$ \\ ${ }^{1}$ Division of Metrology and Optoelectronics, Poznan University of Technology, 3a Piotrowo Street, 60-965 Poznan, Poland
}

\begin{abstract}
The article presents example detector matrices used in long wave thermographic cameras facilitating observations of elements enclosed in SMD housings. Infrared radiation detector matrices parameters and an optical system used in modern long wave thermographic cameras are discussed. The criteria for selecting camera parameters and their impact on the performed measurement are considered.
\end{abstract}

\section{Introduction}

Obtaining the best possible temperature distribution visualisation for the surface of a surface mounted housing (SMD) requires the use of a thermographic camera equipped with an optical system and a matrix of detectors with appropriate parameters. Modern cameras feature an optical system (objective), a fixed FPA matrix comprising IR detectors and electronic amplification, processing and visualisation systems $[5,6,13]$.

\section{Spectral scope}

The first parameter to be considered is the spectral range, within which the FPA matrix operates. Matrices operating in the MWIR ( $3 \mu \mathrm{m}$ to $6 \mu \mathrm{m})$ and LWIR $(6 \mu \mathrm{m}$ to $15 \mu \mathrm{m})$ ranges are used most commonly [1]. This stems from the expected SMD housing surface temperature ranges. The housing temperature depends on the semiconductor junction temperature inside it and the ambient temperature. According to the authors' works, one should expect to observe temperatures in the $20^{\circ} \mathrm{C}$ to $160^{\circ} \mathrm{C}$ range, which corresponds to the $6.69 \mu \mathrm{m}-9.89 \mu \mathrm{m}$ spectral range. It worth noting that this range spans the LWIR and MWIR threshold. The operating spectral range for the detectors making up the matrix depends on the detector type and material which it has been made out of.

\section{IR and NEDT detectors}

Single detectors used for observing SMD housings can be split into two groups: cooled and uncooled operating in ambient temperatures. Detectors with cooling take advantage of quantum effects to absorb infrared radiation. These can be categorised according to the material the semiconductors subsequently used to produce them are made of, into QWIP, MCT and T2SL detectors. The QWIP detector is a quantum well infrared radiation photodetector. It makes use of the quantum well effect in the structure of the two semiconductor bands with different bandgap levels. It mainly comprises AlGaAs and GaAs bands. The operating principle of an MCT detector is different. During its operation, minority carriers are moved by an external electrical field towards the signal electrode. Detectors made out of a number or MCT layers are often found in modern matrices [2]. A T2SL detector is made out of alternate $\mathrm{GaSb}$ and InAs semiconductor bands. Its operation is based on intra band carrier movement and tunnelling though a mulitiband quantum mechanical system [3].

Detectors without cooling (microbolometric) constitute a separate group of detectors. The operation of such a detector is based on a change of resistance as a function of temperature. Modern bolometric detectors comprise an absorber band absorbing the infrared radiation stream reaching the detector, a thermometer and thermal insulation.

NEDT thermal resolution is the last parameter. It determines the smallest detectable temperature difference between given points. For modern thermographic cameras, this value reaches $10-20 \mathrm{mK}$ if using cooled quantum detector matrices and $40-100 \mathrm{mK}$ if using uncooled bolometric detector matrices [2]. A matrix, together with an electronic system for reading and generating a signal is located in a vacuum housing with an objective, providing thermal insulation for the detector from its environment. This delivers high sensitivity and prevents the occurrence of temperature drift $[2,4,5]$.

\section{Optical system}

The objective in a thermographic camera should only allow infrared rays to pass, damping visible spectrum radiation. That is why objectives used in thermography are made out of $\mathrm{Ge}, \mathrm{Si}, \mathrm{ZnSe}, \mathrm{ZnS}$ single crystals and chalcogenide glass. Additionally, in order to increase IR radiation passage and VIS radiation damping, objectives are AR coated and may include bandpass filters. Sometimes special abrasion resistant anti-reflective 
coatings are used with the stricture of a diamond, referred to as hard carbon.

To observe microelements in electronic systems, an objective is needed which will deliver high resolution $(<1 \mathrm{~mm})$ images of objects a few millimetres in size. It should feature a low IFOV value, appropriate geometric resolution and its focal range $f$ should not be larger than the object - detector distance. Narrow-angle objectives, with $\mathrm{f}$ up to $50 \mathrm{~mm}$ (e.g. microscope, with an immersion lens) satisfy these requirements.

Most thermographic cameras feature a built-in objective, however in many cases it can be replaced or an additional one can be mounted. Additionally, modern objectives employ arrays of concave and convex lenses in order to eliminate defects such as spherical aberration or coma. [6] Intermediate rings may also be used to reduce MOD, minimum object distance. Unfortunately, heating up of the ring during a thermographic measurement results in an increase to the measured thermographic value and image distortion, and the longer the ring, the greater this effect. The effect may be reduced using software based non homogeneity correction, but this requires prior measurements using a temperature standard [7].

\section{Comparison of parameters between selected thermographic cameras}

Most thermographic cameras designed for observing microelements are equipped with an uncooled microbolometric matrix, and most often their spectral range spans from $7.5 \mu \mathrm{m}$ to $14 \mu \mathrm{m}$. The spectral range of cameras with a QWIP or MTC detector is significantly lower, for example the FLIR Titanium SC7700L camera (8.1-8.7 $\mu \mathrm{m})$ or the FLIR SC7750L camera $(8.0-9.4 \mu \mathrm{m})$, which reduces the camera's usability for certain applications $[8,9]$. Whereas the spectral range of cameras with a cooled T2SL detector spans both MWIR, as well as LWIR, with lowest-order bounds wavelength of more than $9 \mu \mathrm{m}$ (e.g. Pelican-D LW, $9.3 \mu \mathrm{m}$ ), and as such these can be useful for measurements at the boundary of the medium and long waves [10].

The thermal sensitivity of the available cameras stands at a few dozen or so $\mathrm{mK}$ ( $40 \mathrm{mK}$ on average). Here only the VarioCam HD Research $900(20 \mathrm{mK})$ camera, FLIR SC7000 (20-30 mK) series cameras or FLIR SC660 and FLIR T540 (30 mK) cameras stand out, however the difference in NEDT values is not that significant $[8,11,12]$.

The measurement range for cameras with microbolometric matrices is quite broad, it spans temperatures from $-40^{\circ} \mathrm{C}$ up to as much as $+2000^{\circ} \mathrm{C}$. Here, the FLIR ETS320 camera is noteworthy, with a narrower measurement range (from $-20^{\circ} \mathrm{C}$ to $+250^{\circ} \mathrm{C}$ ), however one which is dedicated to measure temperature distributions in electronic systems (it has a special stand with adjustable object - objective distance) [11]. Whereas the measurement uncertainty for the available cameras stands at $\pm 2{ }^{\circ} \mathrm{C}$ measurement or $2 \%$ of the measured value $\left( \pm 1^{\circ} \mathrm{C}\right.$ or $1 \%$ only sometimes $)$, which today is a standard of a kind when it comes to thermographic camera measurements.

\section{Summary}

A thermographic camera with an objective and a matrix of detectors featuring appropriate parameters should be chosen to observe temperature distributions on SMD housing surfaces. The spectral range, within which detectors in the matrix receive radiation, should overlap as much as possible with the spectral range of radiation emitted by SMD elements' housing surfaces. Furthermore, the smaller the dimensions of an observed object, the more detectors should the used matrix hold. The NEDT value should not be overlooked - it should be as small as possible. On the other hand the selection of an appropriate objective impacts both the sensitivity and spectral range of a camera, as well as its image resolution. The materials which lenses are made out of determine the IR radiation transmission and VIS radiation absorbency. Whereas the focal length, FOV and IFOV parameters determine the minimum size of the observed area and the dimensions of the smallest object which a camera is able to distinguish.

\section{References}

1. K. Chrzanowski, K Firmanty, J. Bareła, PAK 7-8, 34-36 (2005)

2. B. Więcek, Wybrane zagadnienia współczesnej termowizji $w$ podczerwieni (Politechnika Łódzka, Instytut Elektroniki, 2010)

3. http://www.ir-nova.se/t2sl// [07.01.2016]

4. H. Madura, T. Sosnowski, G. Bieszczad, Prz. Elektrotech. 9, 5-8 (2014)

5. W. Minkina, P. Rutkowski, W.A. Wild, PAK 1,. 1114 (2000)

6. M. Vollmer, K-P. Möllmann, Infrared Thermal Imaging. Fundamentals, Research and Applications, (Willey-VCH, 2018)

7. M. Kałuża, PAK 10, 1238-1241 (2011)

8. http://www.flirmedia.com [19.01.2017]

9. http://www.hoskinscientifique.com [19.01.2017]

10. P.C. Klipstein, E. Avnon, Y. Benny et al., Proc. SPIE 9451, 94510K (2015)

11. http://www.flir.com [19.01.2017]

12. http://www.infratec-infrared.com [19.01.2017]

13. A Hulewicz, Elektronika - konstrukcje, technologie, zastosowania 7, 107-110 (2012) 